\title{
Research on the Precise Poverty Alleviation Index System Based on Factor Analysis in Guizhou Province
}

\author{
Hongmei Zhang ${ }^{1,2,3}$ Yongmei $\mathrm{He}^{1,2,3}$ \\ ${ }^{1}$ Guizhou University of Finance and Economics, Institute of Finance \\ ${ }^{2}$ Guizhou Institution for Technology Innovation \& Entrepreneurship Investment \\ ${ }^{3}$ Guizhou Institute of Urban Economics and Development Guiyang Guizhou 550025, \\ China
}

\begin{abstract}
Guizhou Province, as the country with the largest population of poverty, the largest area of poverty and the deepest poverty, has been paying close attention to its return to poverty effect. As of 2016, Guizhou, Yunnan, Guangxi and Guizhou rocky desertification areas (45 counties), Wumeng Mountain area (10 counties) and Wuling Mountain area (16 counties) belong to areas with special difficulties in contiguous rural areas and are crucial targets for poverty alleviation in the new era The main battlefield.In this paper, the 17 key poverty-stricken counties in the contiguous destitute areas are selected as the research objects. The data of 2016 full year, which are calculated in early 2017, are used to construct the index system by using factor analysis. The paper aims at the poverty alleviation and poverty alleviation effects in Guizhou Province, In order to perfect the poverty alleviation management system and promote accurate and efficient poverty alleviation and provide policy recommendations.
\end{abstract}

Keywords

Precise Poverty Alleviation; Factor Analysis; Poverty Reduction; Policy Suggestions

\section{基于因子分析的贵州省精准扶贫评价指 标体系研究}

\author{
张红梅 $1,2,3$ 何永梅 $1,2,3$ \\ 1 贵州财经大学, 金融学院 \\ 2 贵州科技创新创业投资研究院 \\ 3 贵州城镇经济与发展研究院, 贵州, 贵阳 550025
}

摘要：贵州省作为全国贫困人口最多、贫困面积最大、贫困程度最深的省份，其返贫效应一 直备受关注。截止 2016 年，贵州省滇桂黔石漠化区（45 个县）、乌蒙山区（10 个县）、武 陵山片区（16 个县）属于农村集中连片特殊困难地区，是新时期扶贫攻坚的主战场。本文 选取上述连片特困地区中的 17 个重点贫困县作为研究对象, 选取 2017 年初统计的 2016 全 年数据运用因子分析法构建指标体系，旨在针对贵州省贫困及减贫效应，探讨精准扶贫实践 
中的问题及影响因素, 为健全精准扶贫管理体制, 促进精准扶贫高效、有序进行提供政策建 议。

关键词：精准扶贫；因子分析；返贫效应；政策建议

\section{1. 引言}

党的十八大以来, 党中央提出 “精准扶贫” 的思想, 并且在多次重 大会议中强调 “精准扶贫” 的重要战 略思想，把扶贫开发工作纳入 “四个 全面” 战略布局, 大力实施精准扶 贫, 推动贫困地区和贫困群众加快脱 贫致富奔小康的步伐。精准扶贫工作 的高效、有序进行为全面建成小康社 会、消除贫困提供了重要保证。

\section{2. 文献回顾}

\section{1. 国外研究现状}

国外对于贫困的研究最早起源于 1910 年出版的《贫困: 城镇生活研 究》, 该书的作者是英国的学者特朗 里, 在本书中, 特朗里将贫困定义 为: “家庭收入不足以支付维持家庭 成员的最低生活必须开支的状态”。 这种定义将贫困的研究范围仅限定于 ${ }^{1}$

基金项目: 本文由贵州财经大学校级科研 项目（2017XYB07）资助，项目名称：科技 金融支撑贵州精准扶贫的路径与对策研 究。

作者简介: 张红梅, 教授、硕士生导师, 任职于贵州财经大学金融学院, 研究方 向：金融与区域发展、创业投资、风险分 析与管理。Email：754560989@qq. com。何 永梅, 女, 贵州财经大学金融学院研究生 硕士。贵州财经大学在读研究生, 研究方 向：金融与区域发展、创业投资、风险分 析与管理。Email: 411740421@qq. com
家庭生活的开支被认为是收入贫困, 也称为物质贫困。

国外对于金融扶贫的模式，也进 行了一些富有成效的研究。Calvin. Miller(2004) 认为金融市场具有脆弱 性、封闭性、规范程度不高等主要问 题，小贷款公司在参与农村金融扶贫 时所面临的风险较大，同时农业生产 存在弱质性，这些都会在农村金融扶 贫过程当中变为非常棘手的问题。 $\mathrm{Hu}$ Bangyong(2011)对影响金融市场效率的 七个因素通过计量经济学方法建立两 种模型进行分析; DacidevPettenella, MauroMasiero(2014)研究了罗马尼亚金 融服务供给者与农户之间的关系，通 过对农民收入、贷款的使用情况进行 定量分析, 证明贷款在促进农民收入 增长方面的作用比较显著。

\section{2. 国内研究现状}

国内对金融扶贫的研究起步较 晚, 20 世纪 90 年代以来, 权力贫困 (entitlement poverty)的概念自我国开始 被大量提出, 洪朝辉(2003)认为权力贫 困状态下，社会一部分群体的政治、 经济、文化等权利缺少基本的保障, 社会的不平等状态使部分成员由于缺 少一些基本的权利而处于边缘化的界 定, 我们可以通过分析前任的研究成 功对其进行一个比较深入的分析, 来 解释金融扶贫的含义。王晓敏(2009)认 为我国目前的农村财政扶贫政策存在 很多问题，制约了农村扶贫开发进程 的推进。孙璐(2015)以扶贫开发项目绩 效评估为研究对象借助比较分析、系 统分析方法明确扶贫开发项目绩效评 估的关键所在、构建扶贫项目绩效评 
估系统研究框架, 并进一步改进扶贫 项目绩效评估体系, 以适应精准扶贫 背景下扶贫项目推进的新要求。根据 以上国内外研究综述, 可以看出, 目 前国外对于精准扶贫的研究相对成 熟, 其视角和研究方法对国内研究都 具有很好的借鉴作用。因此，本文站 在精准扶贫的视角, 研究如何构架有 效的指标体系和采纳实用的评估方 法, 对扶贫项目进行科学的评估。

\section{3. 因子分析法}

因子分析是用来寻找那些隐藏在 可测变量中的, 无法直接观察到的, 却影响或支配可测变量的潜在因子, 并估计潜在因子对可测变量的影响程 度及潜在因子的关联性的一种多元统 计分析方法。

因子分析的基本原理是分类观测 变量, 将联系比较密切的变量归为同 一类。每一种类的变量被看作一个公 因子，用来表达众多指标或因素之间 的关联关系, 即将每个包含几个联系 比较紧密的变量的类变量作为一个因 子, 然后通过这几个少数的具有代表 性的类因子去传达原始资料当中的多 数信息量。当求出的主要因子解得到 的各主要因子的代表变量不很突出 时, 需要借助适用的因子旋转得出较 为满意的主因子解。最常用的因子旋 转是通过最小方差正交旋转法, 因子 分析模型建立后, 通过应该该模型评 价整体当中每个样本的作用和地位, 做出综合性的评价。对于所研究的问 题就可视降维, 用最少个数的所谓因 子的线性函数与特殊因子之和来描述 原来观测的每一组变量。

\section{1. 因子分析的方法步骤}

第一步为指标的同向化处理, 原 始数据的标准化包括指标正向化和无
量钢化处理两方面。本文采用的方法 是正向化方法。

第二步为指标的标准化处理, 经 过同向化处理的指标还存在各个指标 量纲不同的问题，本文所采取的指标 有百分比形式表示的指标（城镇化 率、贫困人口参加农村合作医疗比例 等) 对各指标数据采取标准化处理方 式如下:

$$
S_{x_{i j}}=\frac{x_{i j}-\overline{x_{j}}}{\sigma_{j}}
$$

第三步为变量指标的相关性检 验, 本文运用 $\mathrm{KMO}$ (Kaiser-MeyerOlkin measure of sampling adequacy) 测 度和巴特莱特球体检验 (Bartlett test of sohericity)检验变量之间的相关性。只 有当各变量的指标通过上述检验时, 因子分析才能真实有效。

第四步为计算相关矩阵 $\mathrm{R}$ 的特征 值和特征向量, 根据特征方程 $|R-\lambda E|=0$

$\lambda$ 及对应的特征向量 $\mathrm{A}, \lambda$ 的大小描 述了各个因子在解释对象所起的作用 的大小。

第五步为计算因子贡献率及累积 贡献率, 确定公共因子个数, 因子贡 献率表示每个因子的变异程度占所有 因子变异程度的比率, 公式为:

$$
C_{i}=\lambda_{i} / \sum_{i=1}^{p} \lambda_{i}
$$

$C_{i}$ 表示方差贡献率。当累积贡献 率达到 $70 \%$ 以上或者特征根不小于 1 , 即确定了公因子的个数。

第六步为求解初始因子载荷矩 阵, $X=A F$, 因子载荷矩阵 $\mathrm{A}$ 并不 唯一, 软件则是运用不同的参数估计 方法求出相应的估计矩阵。本文采用 的方法是主成分法。

第七步为因子载荷矩阵的旋转, 若载荷因子较为平均, 初始的因子载 
荷矩阵描述的经济含义不太明显, 难 以判断各个因子的关系时, 就需要进 行因子旋转。本文采用的是正交旋转 法中的方差最大化旋转。

第八步为计算样本的综合得分, 通过因子载荷矩阵, 可以得出因子得 分系数矩阵 $B$, 然后计算出每个因子的 得分, 最后以各因子的方差贡献率占 因子总方差的贡献率的比重作为权重 加权汇总, 得到应变综合得分。计算 公式为:

$$
F=\frac{\lambda_{1}}{\sum_{i=1}^{m} \lambda_{i}} F_{1}+\frac{\lambda_{2}}{\sum_{i=1}^{m} F_{2}} F_{2}+\ldots+\frac{\lambda_{m}}{\sum_{i=1}^{m} \lambda_{i}} F_{m}
$$

\section{4. 精准扶贫的评价指标体系构建}

在绝大部分贫困地区, 农户的致 贫原因并不是单一的, 在精准扶贫的 帮扶措施上, 往往需要深入分析贫困 户的贫困原因，制定有针对性的帮扶 政策。本文将贵州省精准扶贫减贫效 应指标分为两类：第一类为政策减贫 指标，第二类为社会减贫指标。政策 减贫是指由于政策措施而取得的减贫 效应, 本文选取了连片贫困地区中 “减贫脱帽”乡镇的个数。社会减贫 指标包含农村常住居民可支配收入、 新增通村公路里程、新增 $3 \mathrm{G}, 4 \mathrm{G}$ 基 站、新建安全饮水处、城镇化率、贫 困人口参加农村合作医疗比例、农村 转移城镇人口、新建乡镇污水处理 厂、城镇新增就业人等 9 个指标, 社 会减贫反应了政府从公共基础设施、 经济发展、医疗卫生、环境优化等方 面扶贫的效应。

本文基于指标选取的重要性、可 行性、可比性、科学性等原则, 以 2017 年初统计的 2016 全年贵州省连片 贫困区滇桂黔石漠化区、乌蒙山区和 武陵山区中的 17 个重点贫困县中的经 济指标数据作为样本。

\section{5. 贵州省精准扶贫实证分析}

贵州省滇桂黔石漠化区、乌蒙山 区和武陵山区属于集中连片特困地 区，其中导致这些地区贫困的原因包 含地形上的自然因素、环境污染严 重、以及社会保障、公共基础设施等 多方面。由于地理因素和环境因素的 度量存在一定的难度, 因此本文主要 选取除地理因素和环境因素以外的其 他因素作为参考指标, 由于数据的可 获得性、同一指标的可比性等原因, 本文选取以县为单位进行研究。本文 的数据来源于 2017 初统计的各县的 《2016年政府工作报告》、《贵州省 2016 统计年鉴》等。

下面以 17 个贫困县的数据为分析 数据, 采用 SPSS 24.0 软件作为数据分 析软件。在对精准扶贫指标评价体系 进行因子分析前, 需要检验各变量之 间的相关系数是否满足因子分析的条 件。本文采用常用的 KMO 和 bartlett 形检验。KMO 值反映了各变量之间的 共同因素, 值越大, 表明共同因素越 多。当 $\mathrm{KMO}$ 值 $<0.5$ 时, 表明研究的变 量之间相关性较小, 不适合使用因子 分析。巴特利特球形检验的原假设为 各变量指标之间的相关系数为单位矩 阵, 若巴特利特球形检验的卡方统计 值显著性 $<0.05$, 则表明不能拒绝原假 设, 各变量指标之间的相关性较小, 不适合使用因子分析。KMO 测度值和 Bartlett 球形检验结果如表 1 所示:

表 1 KMO 测度和 Bartlett 球形检验

\begin{tabular}{llr} 
KMO 取样适切性量数。 & .518 \\
\hline 巴特利特球形度检 & 近似卡方 & 62.503 \\
\cline { 2 - 3 } 验 & 自由度 & 45 \\
\cline { 2 - 3 } & 显著性 & .043 \\
\hline
\end{tabular}

根据表 1 分析结果分析, KMO 测 度值为 $0.518>0.5$, bartlett 球形检验的 
近似卡方值为 $62.503, \mathrm{sig}$ 值为 $0.043<0.05$, 说明各变量之间存在相关 关系, 适合做因子分析。本文采取主 成分分析法提取因子，对于个别缺失 的数据, 本文采用平均数进行补充。 由于原数据均为正向指标, 因此直接 进行标准化处理。

本文通过特征值准则，即提取特 征值 $>1$ 的共同因素和碎石图以及累积 方差贡献率来确定提取公因子的个 数, 表 2 反映了所提取的公因子方差 贡献率和累积方差贡献率:

表 2 解释的总方差

\begin{tabular}{|c|c|c|c|c|c|c|}
\hline \multirow[b]{2}{*}{ 成 } & \multicolumn{3}{|c|}{ 初始特征值 } & \multicolumn{3}{|c|}{ 提取载荷平方和 } \\
\hline & & 方差百 & & & 方差百 & \\
\hline 分 & 总计 & 分比 & 累积 \% & 总计 & 分比 & 累积 \% \\
\hline 1 & 2.979 & 29.793 & 29.793 & 2.979 & 29.793 & 29.793 \\
\hline 2 & 2.392 & 23.925 & 53.718 & 2.392 & 23.925 & 53.718 \\
\hline 3 & 1.640 & 16.405 & 70.122 & 1.640 & 16.405 & 70.122 \\
\hline 4 & .977 & 9.771 & 79.893 & & & \\
\hline 5 & .738 & 7.384 & 87.277 & & & \\
\hline 6 & .433 & 4.328 & 91.605 & & & \\
\hline 7 & .305 & 3.046 & 94.651 & & & \\
\hline 8 & .256 & 2.555 & 97.206 & & & \\
\hline 9 & .180 & 1.801 & 99.007 & & & \\
\hline 10 & .099 & .993 & 100.000 & & & \\
\hline
\end{tabular}

提取方法：主成分分析法。

表 2 显示所提取的三个公共因子 的累积方差贡献率为 $70.122 \%$, 达到了 $70 \%$ 的解释标准, 因此, 分析结果达到 了降维的目的。

运用最大方差法(Varimax)对因子 载荷进行旋转, 旋转后的 3 个因子的 成分结果如表 3 所示:
表 3 旋转成分

\begin{tabular}{|c|c|c|c|}
\hline \multirow[b]{2}{*}{ 评估指标 } & \multicolumn{3}{|c|}{ 成分 } \\
\hline & 1 & 2 & 3 \\
\hline “减贫脱帽”乡镇个数 & .434 & -.364 & .658 \\
\hline 农村常住居民可支配收入（元） & .907 & -.012 & .025 \\
\hline 新增通村公路里程 (公里) & -.232 & .787 & -.100 \\
\hline 新建安全饮水处（处） & -.678 & .107 & -.268 \\
\hline 新增 $3 \mathrm{G}, 4 \mathrm{G}$ 基站（个） & -.113 & .087 & .948 \\
\hline 城镇化率 & .813 & -.122 & .048 \\
\hline 贫困人口参加农村合作医疗比例 (\%) & -.245 & -.768 & -.401 \\
\hline 农村转移城镇人口 & .754 & .241 & -.362 \\
\hline 新建乡镇污水处理厂 & -.005 & .823 & -.086 \\
\hline 城镇新增就业人 (万) & -.024 & .595 & -.075 \\
\hline
\end{tabular}

提取方法: 主成分分析法。旋转方法: 凯撒正态化最大方差法。

a. 旋转在 5 次迭代后已收敛。

从表 3 中可以看出, 不同公共因 子在不同变量指标上的因子载荷。根 据各指标变量在三个公共因子上的最 大因子载荷提取三个公因子分别为: 第一类因子，包含农村常住居民可支 配收入（元）、新建安全饮水处 （处）、城镇化率、农村转移城镇人 口，主要反映了贫困地区政策扶持的 减贫效应，这类因子可定义为 “政策 减贫因子”; 第二类因子包含新增通 村公路里程（公里）、贫困人口参加 农村合作医疗比例（\%)、新建乡镇污 水处理厂、城镇新增就业人口, 主要 反映了贫困地区基本的生活条件，可 以定义为 “基本扶贫效应因子” ; 第 三类因子，包括 “减贫脱帽乡镇个 数”、新增 $3 \mathrm{G} \backslash 4 \mathrm{G}$ 基站（个）, 主要 反映了满足生活需求的物质条件, 可 定义为 “高端扶贫效应因子”。

基于本文对 17 个贫困县的精准扶 贫效应进行排序的目的，本文从所提 取的三个公共因子的得分系数矩阵出 发, 以各公共因子的方差贡献率为权 
重建立综合评价模型。从成分得分系 数表中将各变量的标准化数值代入公 式, 以公共因子的方差贡献率为权重 计算综合因子得分，以公共因子对应 的方差贡献率计算 17 个贫困县的精准 扶贫绩效得分及排序, 如表 4 所示: 表 4 综合因子得分

\begin{tabular}{ccc}
\hline 名次 & 地区 & 因子得分 \\
\hline 1 & 普定县 & 2.825018699 \\
\hline 2 & 惠水县 & 1.023824265 \\
3 & 贞丰县 & 0.818128083 \\
\hline 4 & 黄平县 & 0.700214581 \\
\hline 5 & 望谟县 & 0.5624782 \\
\hline 6 & 施秉县 & 0.271969699 \\
7 & 麻江县 & 0.257965296 \\
\hline 8 & 晴隆县 & 0.057110572 \\
\hline 9 & 镇宁县 & -0.346194537 \\
\hline 10 & 荔波县 & -0.533829982 \\
\hline 11 & 台江县 & -0.5938692 \\
12 & 瓮安县 & -0.703627213 \\
\hline 13 & 平塘县 & -0.71167615 \\
14 & 雷山县 & -0.715443266 \\
\hline 15 & 龙里县 & -0.917261761 \\
\hline 16 & 关岭县 & -0.962334902 \\
\hline 17 & 册亭县 & -1.032482383 \\
\hline
\end{tabular}

从综合因子得分排序表中可以看 出, 本文选取的 17 个样本贫困县的精 准扶贫绩效排名, 前三名分别为普定 县、惠水县和贞丰县, 排名最后的三 名分别为龙里县、关岭县以及册亨 县。且从表中可以看出, 排名前 8 位 的县因子得分为正值, 排名后 9 位的 县得分为负值。说明在 2016 全年, 前 8 个县的精准扶贫效果比较显著, 后 9 个县的扶贫效果较前 8 个县差一些。 从三个因子的得分情况来看, 贞丰 县、望谟县、普定县、晴隆县、麻江 县、黄平县在因子的得分较高, 表面 这三个县精准扶贫主要采取了以提高 居民可支配收入（元）、新建安全饮 水处 (处) 、城镇化率、农村转移城 镇人口等政策扶持的方法, 而惠水
县、施秉县主要采取了使农村贫困人 口得到基本生活保障的措施, 而其余 县主要采取了提高贫困人口生活品质 方法, 在这方面成效较为显著。

\section{6. 结论}

本文以贵州省 17 个贫困县精准扶 贫情况为研究对象, 运用因子分析 法, 构建精准扶贫评价指标体系。首 先将通过因子分析找出影响精准扶贫 减贫效应的几方面因素, 并将变量的 因子得分值进行了排序。通过分析发 现, 目前一些贫困县精准扶贫过程中 所采取的措施效果并不显著, 主要原 因可能在于精准识别不到位, 或者没 有对农户的贫困原因进行深入的分 析, 导致帮扶措施不够精准, 因此, 各地区在采取精准扶贫措施时, 应透 过现象看本质, 深入分析农户的致贫 原因, 并进行精准帮扶。以保证精准 扶贫工作高效、有序地进行。

\section{参考文献}

[1] Stefan Dercon: Rural poverty: Old Challenges in New Contexs[J]. The World Bank Research Observer, Cary: Feb 2009. VOL.24.

[2] Christine Farias, Gerard Farias. Cycles of poverty and consumption, the sustainability dilemma[J]. Competitiveness Review, An International Business Journal of Global Competitiveness, 2010(03): 249-250.

[3] 李鲁云, 互联网时代电商扶贫大有 可为 $[\mathrm{J}], 2015(1): 62$.

[4] 全承相, 贺丽君, 全永海.产业扶 贫精准化政策论析 [J]. 湖南财政经 济学院学报, 2015(02).

[5] 凌经球, 赵禹骅.产业扶贫到户:新 阶段扶贫攻坚的重中之重 $[\mathrm{J}]$. 决策 咨询研究, 2014(06). 